\title{
Trivium
}

Revue franco-allemande de sciences humaines et sociales - Deutsch-französische Zeitschrift für Geistesund Sozialwissenschaften

$8 \mid 2011$

Traductions et transferts des savoirs dans l'espace euro-méditerranéen à l'époque médiévale

\section{Übersetzungen und Wissenstransfer}

$\mathrm{Zu}$ einem Aspekt der Beziehungen zwischen lateinisch-christlicher und arabisch-islamischer Welt

Daniel G. König

\section{OpenEdition}

Journals

Édition électronique

URL : http://journals.openedition.org/trivium/3875

DOI : $10.4000 /$ trivium.3875

ISSN : 1963-1820

Éditeur

Les éditions de la Maison des sciences de l'Homme

Référence électronique

Daniel G. König, « Übersetzungen und Wissenstransfer », Trivium [Online], 8 | 2011, online erschienen am 16 Mai 2011, abgerufen am 22 September 2020. URL : http://journals.openedition.org/trivium/ 3875 ; DOI : https://doi.org/10.4000/trivium.3875

Ce document a été généré automatiquement le 22 septembre 2020

\section{(c) (i) () $\Theta$}

Les contenus des la revue Trivium sont mis à disposition selon les termes de la Licence Creative Commons Attribution - Pas d'Utilisation Commerciale - Pas de Modification 4.0 International. 


\section{Übersetzungen und Wissenstransfer}

$\mathrm{Zu}$ einem Aspekt der Beziehungen zwischen lateinisch-christlicher und arabisch-islamischer Welt

Daniel G. König

Was die intellektuellen Wissenschaften angeht,
die dem Menschen natürlicherweise zu eigen
ind, da er ein zum Nachdenken befähigtes Wesen
ist, so sind sie nicht auf eine Gruppe beschränkt,
sondern auf eine bestimmte Geisteshaltung, die
bei allen menschlichen Gruppen anzutreffen ist.
Diese bemühen sich darum, diese
[Wissenschaften], die es in ihrer menschlichen
Form seit der Besiedlung der Schöpfung gibt, zu
verstehen und zu erforschen.
Ibn Hुaldūn (gest. 808/1406)

Die gesellschaftspolitische Dimension eines Forschungsfeldes

\section{Eine Beziehungsgeschichte und ihre Wahrnehmung}

1 Wohl kaum eine Beziehungsgeschichte zwischen Kulturräumen zieht derzeit soviel Aufmerksamkeit auf sich wie die zwischen >dem Westen Gerade hier zeigt sich, wie sehr die Periode, die wir gemeinhin als >das Mittelalter bezeichnen, heutige Diskurse beeinflusst. Einzelphänomene dieser Beziehungsgeschichte sind ein so fester Bestandteil der heutigen Vorstellungswelt, dass sie auch das Bild dieser Beziehungen bis heute maßgeblich prägen. Dies gilt insbesondere für die arabisch-islamische Expansion, die Kreuzzüge und die so genannte 'Reconquistar. Sie beschwören nicht nur Bilder von religiösen Fanatikern herauf, sondern sind - gerade die Kreuzzüge - so stark im konzeptuellen Denken verankert, dass sie für einen geradezu in epische Dimensionen reichenden Antagonismus zweier 
Kulturen stehen, für die eine Variante des Monotheismus (Christentum/Islam) und eine das Geistesleben bestimmende Sprache (Latein/Arabisch) grundlegend sind.

2 Natürlich ist allgemein bekannt, dass es zwischen beiden Kulturräumen auch andersartige Beziehungen gab. ${ }^{2}$ Was mediale Anziehungskraft, konzeptuelle Schlagkraft und Publikumsinteresse angeht, so können sich alternative thematische Zugänge aber kaum mit Themen wie 'ğihā $k$, ,Kreuzzüge` oder >Reconquista messen. ${ }^{3}$ Daher ist verständlich, warum vor einigen Jahren ein internationales muslimisches Autorenkollektiv auf die Notwendigkeit hinwies, die friedlichen Aspekte der Beziehungen zwischen beiden kulturellen Sphären stärker hervorzuheben. Auf diese Weise könnten aktuelle politische Diskurse und damit indirekt auch die Gestaltung der Beziehungen des Westens mit der islamischen Welt positiv beeinflusst werden. ${ }^{4}$

Vor dem Hintergrund des Ideals eines von gesellschaftlichen Diskursen distanzierten und nach Objektivität strebenden Geisteswissenschaftlers ist dieser Aufruf an die Geschichtswissenschaft, >politisch aktiv zu werden, natürlich problematisch. Letztendlich ist es als Fachhistoriker aber kaum möglich, sich aktuellen Diskursen zu Islamismus, Terrorismus, Integrationsfragen oder der heutigen und zukünftigen Rolle des Islam in Europa zu entziehen. Im Bekanntenkreis, aber auch in den Medien und der Politik werden immer wieder Verbindungen zur heutigen Zeit geschlagen, wie etwa die Nutzung des Kreuzzugsbegriffes durch den ehemaligen Präsidenten der Vereinigten Staaten, George W. Bush, vor einigen Jahren deutlich gemacht hat. ${ }^{5}$ Hinzu kommt, dass in zahlreichen Publikationen, die zu öffentlichen Debatten Stellung nehmen, auch historisch argumentiert wird: Häufig wird dabei unter Hervorhebung gewaltsamer Phänomene postuliert, dass die islamische Welt aufgrund der ihr eigenen religiösen Fundamente unfähig sei, Gesellschaften hervorzubringen, die auf den Prinzipien des Rechtsstaates, der universellen Menschenrechte, der Gewaltenteilung und der politischen Partizipation basieren. Die selektive Behandlung bestimmter historischer Ereignisse dient dabei der Untermauerung dieses Postulats. ${ }^{6}$ In diesem Rahmen werden diejenigen, die sich um eine nuanciertere Darstellung der besagten Beziehungsgeschichte bemühen, häufig als naiv belächelt, wenn nicht gar einer Art >Appeasement-Politik ' gegenüber radikalen Islamisten bezichtigt. ${ }^{7}$

\section{Die Gouguenheim-Debatte}

4 In abgeschwächter Form wird dieser Vorwurf auch in dem 2008 unter dem Titel » Aristote au Mont Saint-Michel. Les racines grecques de l'Europe chrétienne erschienenen Werk des französischen Mittelalterhistorikers Sylvain Gouguenheim laut. Ihm zufolge sind der wissenschaftliche und öffentliche Diskurs zum Verhältnis zwischen Europa und der islamischen Welt von der Vorstellung dominiert, dass »einem aufgeklärten, kultivierten und spirituellen Islam ein brutaler, kriegerischer und eroberungslustiger Okzident gegenüberstehe ${ }^{8}{ }^{8}$ Dieser Vorstellung entgegenzutreten ist $u$. a. ein Ziel seines Buches.

5 Dessen eigentliches Thema fasste der Rezensent Roger Pol-Droit in der bekannten Tageszeitung »Le Monde « prägnant unter dem Titel zusammen: »Und wenn Europa sein Wissen nun doch nicht dem Islam verdankte?« Unter anderem an der Tatsache, dass er das Buch als »erstaunliche Korrektur gegenwärtiger Vorurteile« lobte ${ }^{9}$, entzündete sich eine Debatte auf mehreren medialen Ebenen: Lehrende und Studierende von der »Ecole Normale Supérieure Lettres et Sciences Humaines" in Lyon (ENS LSH), Gouguenheims 
Heimatinstitution, distanzierten sich nachdrücklich. ${ }^{10} 56$ renommierte Fachwissenschaftlerinnen und Fachwissenschaftler protestierten in der französischen Tageszeitung "Libération «"11 und veröffentlichten dieses Protestschreiben auch in der arabischsprachigen Zeitung »al-Hayāt «. ${ }^{12}$ Gegenstand einer Tagung an der Sorbonne ${ }^{13}$, einer Podiumsdiskussion auf den angesehenen »Rendez-vous de l'histoire « in Blois ${ }^{14}$ sowie einer weiteren Tagung an der ENS LSH in Lyon ${ }^{15}$ war das Buch Thema von Artikeln und Rezensionen auch in der internationalen englisch-, deutsch-, italienisch- und arabischsprachigen Presse ${ }^{16}$ und provozierte schon zwei Gegenschriften. ${ }^{17}$ Im Rahmen dieser Debatte wurde Gouguenheim aber auch in Schutz genommen: Der renommierte Mediävist Jacques Le Goff soll die Vehemenz der Kritik beklagt haben ${ }^{18}$, rechtskonservative Internet-Blogs deklarierten die Petition von Gouguenheims Heimatinstitution $\mathrm{zu}$ einer fatwa<. ${ }^{19}$ Angesichts solcher Reaktionen warf der Aristoteles- und Averroes-Spezialist Alain de Libera Gouguenheim vor, durch seine banalisierende Vereinfachung bisheriger Forschungsleistungen Bloggern mit rechter Tendenz die Legitimation gegeben $\mathrm{zu}$ haben, aggressiv über eine weitaus differenzierter denkende Fachwelt herzufallen. Dieser würden nun eine »irrationale Liebedienerei« gegenüber dem Islam, »arabophile Gehirnwäsche« und - in Bezug auf ihr Verhalten gegenüber Gouguenheim - "neostalinistische« Methoden vorgeworfen. ${ }^{20}$

6 Dass eine so erhitzte Debatte um das Buch eines Mittelalterhistorikers geführt wird, mag zunächst erstaunen. Denn das eigentliche Thema des Buches - eine kulturhistorische Bewertung der großen Übersetzungsprozesse vom Griechischen und Syrischen ins Arabische vom 8. bis 10. Jahrhundert sowie vom Arabischen ins Lateinische etwa ab dem 12.Jahrhundert - erscheint zunächst weit entfernt von aktuellen gesellschaftspolitischen Debatten. Mit seinem Buch hat Gouguenheim es allerdings geschafft, dieses bisher eher im öffentlichen Abseits stehende Thema zum Zankapfel einer Diskussion zu machen, in der auch um die Deutungshoheit über den Charakter der Beziehungen zwischen sdem Westen` und >der islamischen Welt gerungen wird.

7 Dies liegt unter anderem daran, dass Gouguenheim, wie ja schon der Titel seines Buches impliziert, nach den seigentlichen geistigen Ursprüngen Europas sucht und damit letztlich eine Debatte um die kulturelle Identität Europas führt ${ }^{21}$, ohne dabei sein Europakonzept kritisch $\mathrm{zu}$ reflektieren.22 Vielmehr argumentiert er, dass das 'griechische Erber die eigentliche Wurzel einer unter den Schlagwörtern Gedankenfreiheit, Selbstkritik, Freiheit und Vernunft zusammenfassbaren europäischen Kultur sei ${ }^{23}$, ein Bemühen, das auch in den Schriften anderer Wissenschaftler nachweisbar ist. ${ }^{24}$ Wie auch der Althistoriker Egon Flaig verbindet Gouguenheim die Frage nach der kulturellen Identität Europas mit der Frage nach dessen Verhältnis zur islamischen Welt. ${ }^{25}$ Dabei ist eindeutig festzustellen, dass es ihm darum geht, die islamische Welt als konstruktiven Faktor aus der europäischen Geistesgeschichte auszuschalten:

8 Zum einen minimiert er die Rolle der arabisch-islamischen Welt bei der Vermittlung des griechischen Erbes, indem er einerseits die Bedeutung muslimischer Akteure für diesen Prozess stark reduziert, andererseits die Rolle orientalischer Christen für den griechisch-(syrisch-)arabischen Übersetzungsprozess ${ }^{26}$ sowie die eigenständige Leistung mittelalterlicher europäischer Christen bei der Suche, Übersetzung und Assimilierung dieses Erbes hervorhebt. ${ }^{27}$ Zum anderen behauptet er, dass die arabischislamische Welt aufgrund der Unvereinbarkeit von Islam und griechischer Philosophie 
an den geistigen Errungenschaften Griechenlands wenn überhaupt nur sehr geringen Anteil habe, da es diese trotz aller Übersetzungen nur oberflächlich und ohne tieferes Verständnis assimiliert habe. ${ }^{28}$ Angesichts seiner Überzeugung von einer snur ansatzweise gelungenen Hellenisierung der islamischen Welt wird für Gouguenheim das für die griechische Philosophie charakteristische Streben nach freiem Denken und Rationalität zum dominanten Charakteristikum allein der europäischen Kultur. Europas kulturelle und wissenschaftliche Entwicklung bezeichnet er als »endogen bedingt « und unabhängig von arabisch-islamischen Einflüssen. ${ }^{29}$

\section{Wissenstransfer und transkulturelle Verflechtungen im mittelalterlichen Euromediterraneum}

Mit Gouguenheims Buch erreicht die gesellschaftspolitische Debatte um den Charakter der Beziehungen zwischen `dem Westen und `der islamischen Welt « damit auch einen Bereich, der bisher für das fruchtbare Zusammenwirken von Repräsentanten verschiedener Kulturtraditionen, Religions- und Sprachgruppen stand und damit den Gegenpol zu einer kreuzzugsdominierten Wahrnehmung der historischen Beziehungen zwischen beiden Kulturräumen lieferte: den Bereich der Geisteskultur, der Wissenschaftsgeschichte und des Wissenstransfers, d. h. die Themen dieser Ausgabe.

\section{Verschiedene Dimensionen von Wissenstransfer: Eine Forschungsskizze}

Das Thema des Wissenstransfers vom hellenistischen in den arabisch-islamischen und den lateinisch-christlichen Kulturraum ebenso wie die Frage nach dem arabischislamischen Einfluss auf Europa wurden bisher nicht nur in den plakativen und einseitig ausgerichteten Werken einer Sigrid Hunke oder eines Juan Vernet unter ihren aussagekräftigen Titeln wie »Allahs Sonne über dem Abendland « oder »Was Europa dem spanischen Islam verdankt « behandelt. ${ }^{30}$ In Westeuropa erfreut sich dieser Forschungszweig seit dem 19. Jahrhundert einer Beliebtheit unter philologisch versierten Spezialisten. Dies wird u. a. in zahlreichen, vom »Institut für die Geschichte der arabisch-islamischen Wissenschaften « an der Johann Wolfgang von Goethe-Universität Frankfurt a. M. herausgegebenen Bänden deutlich: Diese behandeln die Rezeption und Assimilation griechischer und indischer Einflüsse in der arabisch-islamischen Welt sowie die Rezeption und Assimilation dieses kulturellen Erbes über die (natürlich nicht ausschließliche) Vermittlung der arabisch-islamischen Welt in Europa. Einzelne Sammlungen sind den Fachbereichen Medizin, Mathematik, Astronomie und Philosophie, andere der Vermittlungsleistung und Rezeption bestimmter Gelehrter gewidmet. ${ }^{31}$ Indem sie Artikel vereinigen, die zwischen 1791 und 1965 in allen großen westeuropäischen Wissenschaftssprachen von renommierten Forscherinnen und Forschern verfasst wurden, geben diese Bände einen Einblick in mehr als $150 \mathrm{Jahre}$ westeuropäischer Forschung in unterschiedlichen Nationalkulturen. ${ }^{32}$ Hochqualifizierte zeitgenössische Forscher, deren zahlreiche Werke hier nicht aufgeführt werden können, haben diese Tradition weitergeführt. ${ }^{33}$ Mittels des Vergleichs tausender Manuskriptseiten und unter Anwendung höchst fachspezifischer Sprachkenntnisse der im Euromediterraneum von der Spätantike bis zur frühen Neuzeit verwendeten Wissenschaftssprachen (Griechisch, Syrisch, Arabisch, Hebräisch, Latein) wurden hier 
die vom Quellenmaterial vorgegebenen Spuren zahlreicher komplexer Vermittlungsprozesse nachvollzogen. An diesen waren nicht nur Christen und Muslime, sondern auch zahlreiche Juden an prominenter Stelle beteiligt ${ }^{34}$, ein Aspekt der von Gouguenheim vernachlässigt wird. ${ }^{35}$ Die im Rahmen dieser Forschungen erzielten Ergebnisse sind zu differenziert, als dass sie sich dazu instrumentalisieren ließen, die europäische Kulturgeschichte auf ıwahre kulturelle Ursprünges zurückzuführen.

11 Dem Bemühen, Europas kulturelle Identität einseitig und unter Ausschluss der arabisch-islamischen Welt auf sendogene Faktoren` zurückzuführen, stehen aber auch andere fachwissenschaftliche Ergebnisse entgegen, die deutlich machen, dass die Kulturgeschichte Europas nicht verstanden werden kann, wenn nicht auch kontinuierliche Wechselwirkungen mit anderen kulturellen Sphären in Betracht gezogen werden. ${ }^{36}$ Eine solche Forschung wendet sich gegen die Vorstellung monolithischer Kulturblöcke und widmet sich mit großer Aufmerksamkeit Phänomenen wie dem Kulturtransfer, kulturellen Austauschprozessen und Hybridphänomenen etwa im Bereich der Sprache ${ }^{37}$ und Kunst. ${ }^{38}$ Bei Letzteren handelt es sich also um nicht nur geographisch verortbare Übergangs- und Vermittlungsräume, ohne deren Existenz die Mobilität von Kulturgütern kaum möglich wäre. Zahlreiche Wortentlehnungen aus dem Arabischen, die im Laufe des Mittelalters und der frühen Neuzeit nicht nur in die Sprachen der Iberischen Halbinsel ${ }^{39}$, sondern in alle modernen westeuropäischen Sprachen bis hin ins Deutsche gelangt sind ${ }^{40}$, machen dabei deutlich, in wievielen Lebensbereichen sich der Einfluss einer Kultur auf die andere manifestieren kann.

Hervorgehoben sei dabei die Vielfalt der vermittelten >objektes: Rohstoffe und Artefakte, aber auch Pflanzen, Tiere und sogar Menschen zählen zu den materiellen Kulturgütern. Religiöses, politisches, philosophisches Gedankengut, aber auch angewandtes, praktisches Wissen ebenso wie Emotionen, Wahrnehmungsmuster und ästhetische Erfahrungen zählen zu den immateriellen Kulturgütern, die Teil solcher Austausch- und Transferprozesse sein können. Immer wieder wird allerdings deutlich, dass sich Materielles und Immaterielles kaum trennen lassen, dass die Vermittlung des einen also immer auch die Vermittlung des anderen mit sich bringt. ${ }^{41}$ Wissenstransfer findet damit nicht nur im Rahmen von Übersetzungen, sondern z. B. auch im Zusammenhang mit der Verbreitung materieller Kulturgüter statt, wie neuerdings eine Studie zur Verbreitung des Zuckers im mittelalterlichen Mediterraneum deutlich macht: Mit dem Konsumprodukt verbreiteten sich auch diejenigen Kenntnisse, die zu seiner Produktion, aber auch zu seiner pharmazeutischen und kulinarischen Nutzung notwendig waren. ${ }^{42}$ Wissenstransfer schlägt sich aber auch in Schriftstücken nieder, die die Wahrnehmung sdes Anderen dokumentieren und es erlauben, einen Prozess der Herausbildung von Wahrnehmungsmustern nachzuvollziehen. Letztere speisen sich aus Erfahrungen, die in der konkreten Auseinandersetzung mit >dem Anderen gewonnen wurden, um später - meist über den Umweg vieler Vermittlungsschritte und Zwischeninstanzen - schriftlich fixiert zu werden. ${ }^{43}$

Ein Akzent der Forschung zu kulturellen Austauschprozessen liegt auf der Untersuchung der an diesen Prozessen beteiligten Akteure und ihrer Lebenswelt. ${ }^{44}$ Neben Gesandten, Seeleuten, Kriegern, Mäzenen, Pilgern, Sklaven wären noch viele weitere beteiligte Gruppen aufzuzählen. Eher als nach ihrem Aktivitätsspektrum oder sozialen Status bietet es sich allerdings an, solche Akteure nach Kriterien zu 
untersuchen, die ihren konkreten Beitrag zum Gelingen von Austausch-, Vermittlungsund Rezeptionsprozessen beleuchten. $\mathrm{Zu}$ diesen zählen ihre Fähigkeiten, ihre Möglichkeiten, aber auch ihre Motivation, sich zwischen ethnisch, religiös und sprachlich definierten Gruppen $\mathrm{zu}$ bewegen. Ein weiteres wichtiges Untersuchungskriterium ist auch ihr Grad der Beteiligung an solchen Prozessen. ${ }^{45}$

\section{Übersetzer als Akteure des Wissenschaftstransfers}

Gerade Übersetzern als Vermittlern zwischen verschiedenen Sprachgruppen und sprachlich fixierten Geisteswelten kommt in diesem Zusammenhang eine besondere Bedeutung zu. Sie standen auch schon vielfach im Zentrum historischer Untersuchungen, allerdings hauptsächlich solcher, die die sprachlichen Beziehungen zwischen post-römisch-hellenistischer, arabisch-islamischer und lateinisch-christlicher Welt weitestgehend außen vor lassen. ${ }^{46}$ Gerade für diesen Bereich fehlt daher noch eine umfassende akteurzentrierte Studie. Dies ist $u$. a. auch dem Quellenmaterial geschuldet, das zwar hinreichend Möglichkeiten zum Vergleich textueller Varianten in verschiedenen Sprachen bietet, aber meist sehr viel weniger Informationen zu den beteiligten Übersetzern liefert.

Folglich haben die meisten Untersuchungen zu den Übersetzern wissenschaftlicher Texte vom Griechischen und Syrischen ins Arabische einen philologischen oder geistesgeschichtlichen Ansatz ${ }^{47}$, auch wenn natürlich immer wieder auf die Übersetzer und ihren sozialen Kontext eingegangen wurde..$^{48}$ Anders als Gouguenheim behauptet, ist der Beitrag syrischer Christen zu diesem Wissenstransfer dabei schon immer gewürdigt worden..$^{49}$ Hervorzuheben ist dabei, dass schon die frühe arabisch-islamische Historiographie detailreiche Angaben zur eng mit dem Römischen Reich verbundenen Geschichte des Christentums und seiner institutionellen Ausformungen enthält. ${ }^{50}$ Der im abbasidischen Osten des 8. bis 10. Jahrhunderts zu verortende Wissenstransfer war also nicht nur auf die Vermittlung naturwissenschaftlich-philosophischen Wissens beschränkt, selbst wenn historiographische Werke kaum übersetzt worden zu sein scheinen. ${ }^{51}$

Philologische und geistesgeschichtliche Fragestellungen beherrschen auch den Zugang $\mathrm{zu}$ den $\mathrm{ab}$ dem 12.Jahrhundert verstärkt stattfindenden Übersetzungen vom Arabischen und Hebräischen ins Lateinische. Allerdings finden sich auch hier Studien zu Biographie, gesellschaftlicher Rolle und sozialem Umfeld der beteiligten Übersetzer ${ }^{52}$, so wie auch der Beitrag jüdischer Gelehrter zu diesem Wissenstransfer schon immer als maßgeblich anerkannt wurde. ${ }^{53}$ Neben zahlreichen naturwissenschaftlichen und philosophischen Texten bezeugen die lateinische Fassung des $\operatorname{Koran}^{54}$, die portugiesischen und kastilianischen Versionen der "Crónica del moro Rasis $\aleph^{55}$ sowie die »Historia Arabum « des Rodrigo Jiménez de Rada ${ }^{56}$, dass auch theologische und historiographische Texte übertragen wurden. ${ }^{57}$

Untersuchungen zur Übersetzung lateinischer Texte ins Arabische wiederum sind bisher nicht sehr weit über die Verortung plurilingualer Milieus herausgekommen: Eine große Rolle spielen hier die so genannten mozarabischen Christen der Iberischen Halbinsel, für deren Vielsprachigkeit es nicht an Beweismaterial mangelt ${ }^{58}$ und die deswegen auch Gegenstand linguistischer Untersuchungen geworden sind.$^{59}$ Bei den drei bekanntesten Texten bereitet es allerdings Schwierigkeiten, die Übersetzer zu bestimmen oder genau $\mathrm{zu}$ verorten: Die Übertragung der von Hieronymus 
angefertigten lateinischen Übersetzung der Psalmen durch einen gewissen Ḥaf̦̣ bin Albar al-Qūțī, der u.a. auch eine äußerst interessante Einleitung zu übersetzungstechnischen Fragen vorangeht, kann nicht mit letzter Sicherheit chronologisch verortet werden und wird entweder dem Ende des 9. oder des 10. Jahrhunderts zugeschrieben. ${ }^{60}$ Aufgrund widersprüchlicher Angaben in den Quellen erscheint es nicht möglich, den bzw. die Übersetzer des etwa im gleichen Zeitraum verfassten »kitāb Hūrūšiyūš« endgültig zu bestimmen. ${ }^{61}$ Hierbei handelt es sich um eine von Mayte Penelas als "compilation-cum-translation " bezeichnete ${ }^{62}$ arabische Übertragung der »Historia adversus paganos" des spätantiken Historiographen Orosius (gest. um 417), in die auch zahlreiche andere lateinische Texte eingeflossen sind. ${ }^{6}{ }^{6}$ Über die von dem arabisch-islamischen Gelehrten al-Mas'ūdī (gest. 345/956) genutzte Chronik der Franken, die im Jahre 328/939-940 vom Bischof Godemar von Gerona dem zukünftigen Kalifen von Córdoba, al-Ḥakam II, übergeben worden sein soll, ist schließlich am wenigsten bekannt: Weder wird aus al-Mas'ūdī's spärlichen Angaben deutlich, in welcher Originalsprache die Chronik verfasst, noch ob bzw. wie und wann sie übersetzt wurde. Nur ihre fränkische Herkunft erlaubt die Vermutung, dass es sich dabei ursprünglich um einen lateinischen Text gehandelt haben mag. ${ }^{64}$ In einem ganz anderen Kontext kommt es einige Jahrhunderte später zu einer weiteren Welle von Übersetzungen vom Lateinischen ins Arabische: U. a. mit Unterstützung des Vatikans werden etwa ab dem 16. Jahrhundert sowohl von orientalischen Christen als auch von im Orient wirkenden katholischen Missionaren zahlreiche lateinische theologische Texte ins Arabische übertragen. ${ }^{65}$

\section{Weitere Aspekte sprachbasierter Vermittlungsakte}

Jenseits der Übersetzung von Texten wurde die Rolle sprachlicher Vermittler auch im nichtwissenschaftlichen Kontext untersucht, etwa im normannisch-angevinischen Süditalien ${ }^{66}$, in den Kreuzfahrerstaaten ${ }^{67}$, im Rahmen der missionarischen Aktivitäten von Franziskanern und Dominikanern ${ }^{68}$, im Zusammenhang mit der Eingliederung der kanarischen Inseln in den euromediterranen Kulturraum ${ }^{69}$ etc. Dennoch erscheinen sowohl der Bereich der mündlichen Wissensvermittlung von einer Sprache in die andere als auch das Thema der sprachlichen Übertragung nichtwissenschaftlicher, z. B. rechtlicher Texte noch weitgehend unterbelichtet. Mehrere Fragen stehen dabei zur Debatte:

Zum einen ist oft nicht eindeutig, welcher Umgangssprache sich Repräsentanten der lateinisch-christlichen und der arabisch-islamischen Welt im alltäglichen, aber auch im offiziellen, etwa diplomatischen Kontakt bedienten. Oftmals scheint man auf die im islamischen Herrschaftsgebiet befindlichen religiös definierten jüdischen und christlichen Minderheiten zurückgegriffen $\mathrm{zu}$ haben, um Kommunikation $\mathrm{zu}$ ermöglichen: So werden etwa Juden als Vermittler sowohl in den lateinischen Beschreibungen des Gesandtenaustauschs zwischen Karl dem Großen und Hārūn arRašĩd erwähnt ${ }^{70}$ und auch in dem Werk des persischen Geographen Ibn Hurradadbih (gest. ca. 300/911) als sprachlich versierte Vermittler zwischen fränkischer und abbasidischer Sphäre beschrieben. ${ }^{71}$ Auch noch später, etwa im Rahmen der Gesandtschaft zwischen Otto I. (regn. 936-973) und 'Abd ar-Raḥmān III. (regn. 912-961) scheint der offizielle Informationsaustausch über die Angehörigen religiöser Minderheiten gelaufen zu sein. ${ }^{72}$ 
20 Der alleinige Hinweis auf die durchaus hervorzuhebende Bedeutung dieser Minderheiten und ihrer Sprachkenntnisse wird der Komplexität der Beziehungen zwischen lateinisch-christlicher und arabisch-islamischer welt aber letztlich nicht gerecht. Gerade in den zahlreichen Grenzregionen zwischen lateinisch-christlicher und arabischer Welt ließ sich die Alltagskommunikation nicht überall und allezeit auf Minderheiten abwälzen. ${ }^{73}$ Nicht nur erwähnen selbst die Quellen des Frühmittelalters schon Beispiele für direkte Kommunikation, so etwa - wenn auch in anekdotischer Form - zwischen »Agareni« aus Nordafrika auf der einen, Menschen aus Amalfi und Salerno auf der anderen Seite. ${ }^{74}$ Wir finden auch Beispiele für muslimische Kriegsgefangene, deren im Laufe der Zeit erworbene Position, z. B. als Gehilfen eines Abtes, suggeriert, dass sie im Rahmen ihrer Gefangenschaft die dazu notwendigen Sprachkenntnisse erworben haben. ${ }^{75}$ Das Beispiel des muslimischen Pilgers Ibn Ğubayr (gest. 614/1217) zeigt, dass alltäglicher Kontakt zur Sprachvermittlung beitrug: In den Abschnitten seines Reisetagebuches, die seine Durchquerung der Kreuzfahrerstaaten und seine Fahrt auf einem genuesischen Schiff beschreiben, beginnt er, lateinische Monatsnamen zu notieren ${ }^{76}$, und dokumentiert zudem ein romanisch anmutendes Wort für Pilger (»al-bilǵriyyūn«). ${ }^{77}$ Wie weit die - sicher auch durch die lateinischchristliche Expansion in den Mittelmeerraum geförderte - Assimilierung romanischen Sprachgutes seitens der Muslime gegangen sein muss, wird daran deutlich, dass die im neuzeitlichen Mittelmeerraum nachweisbare »lingua franca» zum größten Teil aus romanischen Sprachelementen besteht. ${ }^{78}$

21 Doch darf der Bereich der Kommunikation über Sprachgrenzen hinweg nicht nur auf hochkomplexe wissenschaftliche Übersetzungen einerseits, die sprachliche Bewältigung von Alltagssituationen andererseits reduziert werden. Spätestens ab dem 12. Jahrhundert ist im Rechtsbereich eine gewisse Institutionalisierung sprachlicher Vermittlungsprozesse zu verzeichnen. Dies lässt sich nicht nur an der Existenz und Fachterminologie von trilingualen Urkunden im normannischen Sizilien ${ }^{79}$ sowie von bilingualen Verträgen zwischen Repräsentanten zahlreicher lateinisch-christlicher und arabisch-islamischer Herrschaftsträger nachweisen. ${ }^{80}$ Sie zeigt sich auch in der Erwähnung von Übersetzern und Dolmetschern: Auf deren Hilfe waren die beteiligten Parteien angewiesen, um ein entsprechendes Rechtsgeschäft auszuhandeln und schließlich in zwei Sprachen schriftlich zu fixieren. Nachweise für deren Aktivität finden sich etwa in den »Siete Partidas« Alfons X. (regn. 1252-1284) von Kastilien-Léon, die die sprachlichen Modalitäten für Vertragsschlüsse innerhalb seines multilingualen Herrschaftsgebietes regeln. ${ }^{81}$ Auch in den Quellen zum Rechtsalltag der Kreuzfahrerstaaten spielen sie eine große Rolle ${ }^{82}$, möglicherweise in Adaptation weit zurückreichender islamischer Vorbilder. ${ }^{83}$ Auch zum Abschluss internationaler Verträge, etwa zwischen den italienischen Seerepubliken und verschiedenen Fürstentümern Nordafrikas oder den Kreuzfahrern und ihren Nachbarn, waren Übersetzer und Dolmetscher notwendig: Die Schwierigkeiten, einen Rechtsinhalt in zwei Sprachen unter Wahrung aller Inhalte und auf elegante Weise auszudrücken, werden u. a. im Sekretärsmanual des in der mamlukischen Kanzlei tätigen al-Qalqaš andī (gest. 821/1418) erwähnt. ${ }^{84}$ Aus einigen Verträgen geht dabei hervor, dass komplizierte Mechanismen entwickelt wurden, die Korrektheit der jeweiligen Übersetzung zu gewährleisten: Der 1313 zwischen Sancho I. von Mallorca und dem Hafsiden Abū Yaḥyā Zakariyā' bin al-Liḥyānī abgeschlossene Vertrag etwa wurde von einem gewissen Johannes Egidius aus dem Arabischen ins Katalanische übersetzt und dabei wohl laut vorgelesen (»legente«) und erklärt (»interpretante et explanante«). 
Daraufhin wurde er von einem Notar des Königs von Aragón ins Lateinische übertragen, die verschiedenen Sprachversionen unter Zuhilfenahme des genannten Dolmetschers und Übersetzers Wort für Wort (»de verbo ad verbum«) gegeneinander geprüft. In diesem spezifischen Fall teilte der Übersetzer dem Notar dann im Auftrag des Hafsidenherrschers und seines juristischen Beraters mit, dass eine bestimmte Klausel nicht in der arabischen Version enthalten sei, aber nachgetragen werde. ${ }^{85}$

Die im so genannten Sprachenkanon des Konzils von Vienne (1311-1312) enthaltene Bestimmung, in Rom, Paris, Oxford, Bologna und Salamanca Lehrstühle für Arabisch, Hebräisch und »Chaldäisch « zu errichten ${ }^{86}$, liefert neben Hinweisen auf des Arabischen mächtige Übersetzer an der päpstlichen Kurie ${ }^{87}$, auf Arabischkenntnisse von Dominikanern ${ }^{88}$ etc. einen Einblick in die Anfänge einer institutionellen Vermittlung orientalischer Sprachen im lateinischen Westen des 13. und 14. Jahrhunderts, deren Förderung v. a. missionarisch motiviert war. Mangels ähnlicher Beweise auf arabischislamischer Seite wird gerne mit Bernard Lewis vermutet, dass die für die Kommunikation mit der lateinischen Christenheit notwendigen Fremdsprachenkenntnisse von Muslimen als unwichtig, wenn nicht gar als verachtenswert erachtet und deswegen v. a. von Nichtmuslimen gepflegt wurden. ${ }^{89}$ In dieser Schärfe gilt es diese These allerdings zu überprüfen: Nicht nur waren an der lateinischen Übersetzung des Koran sowie der portugiesischen Übersetzung der » Crónica del moro Rasis « Muslime beteiligt. ${ }^{90}$ Die italienischen Seerepubliken, die "Sarazenen« zumindest zeitweise ungestörten Aufenthalt in ihrem Territorium garantierten $^{91}$, verfügten auch über Schreiber, deren Namen auf muttersprachliche Arabischkenntnisse und muslimische Religionszugehörigkeit schließen lässt. ${ }^{92}$ Ebenso sind zumindest in einigen Vertragstexten Übersetzer und Dolmetscher im Dienste islamischer Herrscher erwähnt, die sowohl Muslime als auch einer von Europäern gesprochenen oder geschriebenen Sprache mächtig gewesen zu sein scheinen. ${ }^{93}$ Die Tatsache, dass 1421 zwischen dem Hafsidenherrscher Abū Fāris 'Abd al-'Azīz und Vertretern von Pisa und Florenz einvernehmliche Regelungen zu den Verpflichtungen von Dolmetschern gegenüber Kaufleuten sowie zur Bezahlung ihrer Dienste getroffen wurden, suggeriert, dass im Hafsidenreich ein auch außerhalb der europäischen Diaspora stehender Dienstleistungsmarkt vorhanden war, der nicht notwendigerweise von einer ethnischen, religiösen oder sprachlichen Gruppe dominiert wurde. ${ }^{94}$

\section{Zu den Beiträgen dieses Bandes}

Der Wissenstransfer zwischen lateinisch-christlicher und arabisch-islamischer Welt umfasst also zahlreiche Übersetzungsprozesse, teilweise auch monumentalen Charakters. Allerdings sollte auch deutlich geworden sein, dass sich Wissenstransfer nicht auf schriftlich fixierte und dem Bereich der Wissenschaftsgeschichte zuzuordnende Übersetzungsleistungen reduzieren lässt. Wissen wurde zum einen auch ohne die Zuhilfenahme von Sprache ausgetauscht und vermittelt. Zum anderen berührte der Wissenstransfer, selbst wenn er von sprachlicher Vermittlung abhing, auch andere Sphären menschlicher Aktivität. Dieser Vielfalt an Formen kann die gemeinsam mit Yassir Benhima erfolgte Auswahl der nun folgenden Artikel nur ansatzweise gerecht werden. Dennoch lagen ihr bestimmte Kriterien zugrunde, die gewährleisten sollten, dass diese Ausgabe einen Einblick, wenn schon nicht in alle, so doch wenigstens in sehr verschiedene Aspekte des Leitthemas vermittelt: 
Erstens galt es, einen exemplarischen Einblick in die "großen« Übersetzungsprozesse zu geben: Der Beitrag von Gotthard Strohmaier vergleicht die Rezeption der griechischen Wissenschaften im lateinischen Westen des 4. bis 6. Jahrhunderts mit der syrisch-arabischen Rezeption desselben Materials im post-römischen Nahen Osten. Einen tieferen Einblick in die Rolle orientalischer Christen vermittelt dabei der Artikel von Javier Teixidor. Diesen Texten stehen zwei Beiträge zur Rezeption der arabischen Wissenschaftstradition im lateinischen Westen gegenüber: Der Aufsatz von Jacques Langhade beschäftigt sich mit dem Entstehungskontext und der Rezeption der Schriften von Ibn RuŠd / Averroes und behandelt damit eines der wichtigsten Werke des arabisch-lateinischen Wissenstransfers. Um darauf hinzuweisen, dass dieser Transfer weit mehr Wissensbereiche umfasste als die Rezeption aristotelischen Denkens, wurde der gemeinsam von Danielle Jacquart und Gérard Troupeau verfasste Aufsatz zur Übersetzung medizinischer Werke hinzugezogen.

Zum zweiten ging es darum, auch auf die Existenz von Wissenstransfer außerhalb dieser monumentalen Übersetzungsprozesse hinzuweisen. Exemplarisch stehen hierfür die Beiträge von Johannes Pahlitzsch und Anna Akasoy. Ersterer beleuchtet das Zusammenspiel medizinischer Experten unterschiedlicher ethnischer und religiöser Provenienz im Nahen Osten der Kreuzzüge und zeigt u. a. auf, dass Fachwissen durchaus auch außerhalb der textuellen Sphäre vermittelt werden konnte. Die Gegenüberstellung der beiden medizinhistorischen Beiträge von Pahlitzsch und Jacquart/Troupeau macht dabei deutlich, dass selbst in einem Wissensbereich Information an diversen Orten, zu unterschiedlicher Zeit und in verschiedenen Milieus über zahlreiche und voneinander unabhängige Kanäle vermittelt wurde. Der Artikel von Akasoy wiederum soll dazu anregen, nach den impulsgebenden Kräften zu fragen, die den intellektuellen Austausch über kulturelle Grenzen hinweg fördern, verstärken oder gar auslösen. Wie auch im Rahmen der arabisch-islamischen Expansion um das Mittelmeer im 7. und 8. Jahrhundert sowie der lateinisch-christlichen Expansion auf der Iberischen Halbinsel und in den Mittelmeerraum etwa ab dem 11. Jahrhundert jeweils gefolgt von monumentalen Übersetzungsleistungen! -, etablierten sich im Zuge der osmanischen Eroberung Konstantinopels neue Eliten in einem der wichtigsten kulturellen Zentren der mittelalterlichen Welt. Akasoy geht nicht nur auf die intellektuellen Assimilationsprozesse ein, die infolge dieser massiven politischen Veränderungen stattfanden, sondern beschäftigt sich auch mit denjenigen Faktoren, die die Assimilation des byzantinischen Erbes beeinflusst haben. Damit gibt sie Einblick in die Mechanismen komplexer Akkulturations- und Filterungsprozesse, die Teil eines jeden Wissenstransfers sind, aber immer in neuen Varianten verlaufen.

Zum dritten galt es, die Vielfalt der an verschiedenen Formen des Wissenstransfers beteiligten und interagierenden Personen und Gruppen herauszustellen, die vom Römischen Reich der Spätantike bis zum Aufstieg des Osmanischen Reiches zur Vermittlung und Verbreitung von Wissen beitrugen. Mit zwei Artikeln - von Teixidor und Pahlitzsch - sollte dabei der bedeutenden Rolle von ethnisch, religiös und/oder sprachlich definierten Minderheiten Rechnung getragen werden, die - wie schon betont wurde - eine maßgebliche Mediatorenrolle zwischen verschiedenen Kulturräumen einnahmen. Die Vielfalt der dabei behandelten Milieus macht dabei deutlich, dass trotz aller Grenzen und Barrieren jede historische Epoche Menschen hervorbringt, die die Fähigkeit haben, als Vermittler zu fungieren. 

verschiedenen, bei der Rekonstruktion solcher Prozesse des Wissenstransfers verwendeten wissenschaftlichen Methoden zu vermitteln: Vom makrohistorischen und komparatistischen Zugriff Strohmaiers über die gruppenspezifische Analyse von Rezeptionsprozessen bei Teixidor, Pahlitzsch und Akasoy, von der philologischen Untersuchung fachwissenschaftlicher Termini bei Jacquart/Troupeau zur biographisch-rezeptionsgeschichtlichen Darstellung von Langhade werden verschiedene Arten des Umgangs mit einem Thema dargeboten, das aufgrund seines disziplinenübergreifenden Charakters schon immer wissenschaftlich interessierte Menschen unterschiedlicher Art zusammengebracht hat.

Ein letzter Beitrag wurde schließlich ausgewählt, um den Bogen zu schließen und auf die anfangs angesprochene gesellschaftspolitische Relevanz von sprachlichen Vermittlungsprozessen zwischen lateinisch-christlicher und arabisch-islamischer Welt zurückzuverweisen. Der Kommentar Thomas Ricklins zu den Thesen Gouguenheims bietet einen Beitrag zu der anfangs erwähnten Debatte um die Tragweite arabischislamischer Einflüsse auf die europäische Geistesgeschichte und mag frankophonen Leserinnen und Lesern einen Eindruck davon vermitteln, wie das Buch »Aristote au Mont Saint-Michel « in der deutschen Wissenschaftslandschaft aufgenommen wurde.

Abschließend würde ich gerne meinen Dank aussprechen; nicht nur dem wissenschaftlichen Beirat von Trivium, der dem Vorschlag, eine Ausgabe zur behandelten Thematik zu veröffentlichen, zugestimmt hat, sondern auch den beteiligten Autoren und Verlagen für Ihre Unterstützung des Projektes. Mein besonderer Dank geht allerdings an diejenigen, die an der Vorbereitung dieser Ausgabe beteiligt waren: In der Trivium-Redaktion betrifft dies v.a. den Redaktionsleiter Hinnerk Bruhns sowie die ProjektmitarbeiterInnen Cécile d'Albis, Katrin Heydenreich, Bernd Schwibs und Julie Sentis. Ohne ihre Bemühungen - besonders seitens der Redaktionssekretärin Katrin Heydenreich -, wäre diese Veröffentlichung, die das Ergebnis einer fruchtbaren und freundschaftlichen Zusammenarbeit darstellt, nicht zustande gekommen. Zudem bedanke ich mich sehr herzlich bei den Übersetzern Mathieu Olivier, Andreas Pfeuffer, Anne-Laure Vignaux, Frédéric Vitoux und Andreas Wittenburg für ihr Engagement und die Geduld, mit der sie die unvermeidliche Suche nach Kompromissen ertragen haben.

\section{BIBLIOGRAPHIE}

\section{Quellen}

Ademarus Cabannensis, Chronicon, ed. Pascale Bourgain, Richard Landes, Georges Pon, Turnhout, 1999 (Corpus Christianorum, Continuatio medievalis, 129).

al-Mas' ūdī, kitāb at-tanbīh wa 'l-išrāf, ed. Michael J. de Goeje, Leiden 1893. 
al-Mas'ūdī, murūğ adِ-dahab wa ma'ādin al-ǧawhar § 912, ed./übers. Charles Barbier de Ménard, Abel Pavet de Courteille, Charles Pellat, Beirut 1965-1979 / Paris 1962-1997

al-Qalqašandī, kitāb șubḥ al-'ašā', ed. Muḥammad 'Abd Allāh Ibrāhīm, 14 Bde, Kairo, 19151922.

Annales regni Francorum, ed. Georg H. Pertz, Friedrich Kurze, Hannover, 1895 (MGH SS rer. Germ. in us. schol., 6).

Crónica del moro Rasis, ed. Diego Catalán, María Soledad de Andrés, Madrid, 1975.

Ibn Ğubayr, riḥlat Ibn Ğubayr, kein Editor, Beirut, ohne Jahr (Verlag: dār aṢ-șādir).

Ibn Ḩaldūn, tārīh̆, ed. Suhayl Zakkār, Ḩalīl Šaḥāda, 8 Bde., Beirut, 2000-2001.

Ibn Huurradaḍbih, kitāb al-masālik wa'l-mamālik, ed. Michael de Goeje, Leiden, 1889.

Iohannes abbas Sancti Arnulfi, Vita Iohannis Gorziensis, ed. Georg Heinrich Pertz, Hannover, 1841 (MGH SS in folio, 4), S. 335-377.

kitāb Hurūšiyūš (traducción árabe de las historiae adversus paganos de Orosius), ed. Mayte Penelas, Madrid, 2001.

Las Siete Partidas, ed. Robert I. Burns, übers. Samuel P. Scott, Bd. 4 : Family, Commerce, and the Sea. The Worlds of Women and Merchants, Philadelphia, 2001.

Le psautier mozarabe de Hafs le Goth, ed. Marie-Thérèse Urvoy, Toulouse, 1994.

Maçoudi, Le Livre de l'avertissement et de la revision, übers. Bernard Carra de Vaux, Paris, 1897.

Matthaeus Parisiensis, chronica majora, ed. Henry Richards Luards, Bd. 4 (A. D. 1240-1247), London, 1877.

Petrus Venerabilis, Contra sectam Saracenorum, ed. James Kritzeck, in: James Kritzeck, Peter the Venerable and Islam, Princeton, 1964.

Radulfus de Diceto, Opera historica, ed. William Stubbs, Bd. 2, London, 1876 (Rolls Series, 68).

Siete Partidas, ed. La Real Academia de la Historia, Bd. 3, Madrid, 1807.

\section{Sekundärliteratur}

Aillet, Cyrille / Makariou, Sophie / Tixier-Caceres, Emmanuelle / Gourdin, Philippe / MartinezGros, Gabriel (2001): Pays d'Islam et monde latin, 950-1250, Neuilly-sur-Seine.

Aillet, Cyrille / Penelas, Mayte / Roisse, Philippe (Hg.) (2008): ¿Existe una identitad mozárabe? Historia, lengua y cultura de los cristianos de al-Andalus (siglos IX-XII), Madrid.

al-Ḥayāt (2008): »Dayn Ūrubba aț-țaqāfî li'l-‘ālam al-islāmī«, al-Hayāt, 14.05.2008, http:// www.daralhayat.com/archivearticle/203028, heruntergeladen am 13.05.2011.

Altaner, Berthold (1933): »Die fremdsprachliche Ausbildung der Dominikanermissionare während des 13. und 14. Jahrhunderts«, Zeitschrift für Missionsgeschichte, 23, S. 233-241.

Arrignon, Jean-Pierre / Bousquet-Labouérie, Christine / Leroy, Béatrice (2001): Pays d'Islam et monde latin: milieu $\mathrm{X}^{e}$ - milieu XIII ${ }^{e}$ siècle, Paris.

Ashtor, Eliyahu (1977): »Aperçu sur les Radhanites «, Schweizerische Zeitschrift für Geschichte, 27, S. 245-275. 
Aslanov, Cyril (2009): »Joseph Caspi et le plurilingualisme des Juifs provençaux«, in: Kappler, Claire / Thiolier-Méjean, Suzanne (Hg.): Le Plurilinguisme au Moyen Âge. Orient-Occident, Paris, S. 111-122.

Attias, Jean-Christophe (2009): »Judaïsme: le tiers exclu de l'Europe chrétienne«, in: Büttgen / de Libera / Rached / Rosier-Catach (Hg.): Les Grecs, les Arabes et nous, S. 213-222.

Badawī, Aḥmad (1982): Awrūsiyūs. tārīh al-‘ālam, Beirut.

Bakr, Salwa / Ezbidi, Basem / Kassab-Hassan, Hanan / Karcic, Fikret / Zaidi, Mazhar / Jawhar Hassan, Dato' (2004): Der Westen und die islamische Welt. Eine muslimische Position, hg. vom Institut für Auslandsbeziehungen (ifa) Stuttgart, Stuttgart.

Balard, Michel / Bresc, Henri / Durand, Robert (2000): Islam et monde latin (milieu Xe - milieu XIII ${ }^{e}$, Paris.

Bawer, Bruce (2006): While Europe Slept. How Radical Islam is Destroying the West from Within, New York.

Beer, Jeannette M. A. (1989): Medieval Translators and their Craft, Michigan.

Ben Jelloun, Tahar (2008): »Un professore tutto occidentale«, La Repubblica, 23.05.2008, S. 56.

Borgolte, Michael / Schiel, Juliane (2008): »Mediävistik der Zwischenräume«, in: Borgolte, Michael / Schiel, Juliane / Schneidmüller, Bernd / Seitz, Annette (Hg.): Mittelalter im Labor. Die Mediävistik testet Wege zu einer transkulturellen Europawissenschaft, Berlin, S. 15-24.

Borgolte, Michael / Schneidmüller, Bernd (Hg.) (2010): Hybride Kulturen im mittelalterlichen Europa, Berlin.

Bosselmann-Cyran, Kristian (1997): »Dolmetscher und Dragomane in Palästina und Ägypten. Über sprachkundige Galeerensklaven, Renegaten und Mameluken im ausgehenden Mittelalter«, Das Mittelalter, 2(1), S. 47-66.

Buresi, Pascal (2004): La Frontière entre chrétienté et Islam dans la péninsule Ibérique: du Tage à la Sierra Morena (fin $\mathrm{XI}^{e}-$ milieu XIII ${ }^{e}$ siècle), Paris.

Burman, Thomas E. (1994): Religious Polemic and the Intellectual History of the Mozarabs, c. 1050-1200, Leiden.

Burman, Thomas E. (2007): »How an Italian Friar Read His Arabic Qur'an«, Dante Studies, 125, S. 93-109.

Burman, Thomas E. (2009): Reading the Qur'an in Latin Christendom, 1140-1560, Philadelphia.

Burnett, Charles (2009): Arabic into Latin in the Middle Ages. The Translators and their Intellectual and Social Context, Farnham.

Burns, Robert I. / Chevedden, Paul E. (1999): Negotiating Cultures. Bilingual Surrender Treaties in Muslim-Crusader Spain, Leiden / Boston / Köln.

Büttgen, Philippe / de Libera, Alain / Rached, Marwan / Rosier-Catach, Irène (Hg.) (2009): Les Grecs, les Arabes et nous. Enquête sur l'islamophobie savante, Paris.

Canale, Michele Giuseppe (1860): Nuova istoria della repubblica di Genova: Epoca seconda (1190-1270): Il potestà, Florenz.

Christ, Georg (2006): »Masking Cooperation with the Infidel. The Venetian Commercial Privileges, Political Power and Legal Culture in Mamluk Egypt «, in: Cimdina, Asuma / Osmond, Jonathan (Hg.): Power and Culture. Hegemony, Interaction and Dissent, Pisa, S. 33-51. 
CIHAM (UMR 5648) (2000): Pays d'Islam et monde latin (X'-XIII siècle). Textes et documents, Lyon.

Cohn, Willy (1935): »Jüdische Übersetzer am Hofe Karls I. von Anjou, Königs von Sizilien (12661285)《, Monatsschrift für Geschichte und Wissenschaft des Judentums, 79, S. 246-60.

Collectif international de 56 chercheurs en histoire et philosophie du Moyen Age (2008): »Oui, l'Occident chrétien est redevable au monde islamique«, Libération, 30.04.2008, www.liberation.fr/ tribune/010179795-oui-l-occident-chretien-est-redevable-au-monde-islamique, heruntergeladen am 19.11.2010.

Colloque (2009): L'Islam et l'Occident à l'époque médiévale: Transmission et diffusion des savoirs (11.13.03.2009), als Video oder MP3 einsehbar unter: http://www.canal-u.tv/producteurs/ ecole_normale_superieure_de_lyon/dossier_programmes/ colloque_1_islam_et_l_occident_a_l_epoque_medievale_transmission_et_diffusion_des_savoirs/ 001_colloque_1_islam_et_1_occident_a_1_epoque_medievale.

Corriente, Federico (2008): Dictionary of Arabic and Allied Loanwords, Leiden.

Daiber, Hans (1986): »Orosius' Historiae adversus paganos in arabischer Überlieferung«, in: van Henten, J. W. (Hg.): Tradition and Reinterpretation in Jewish and Early Christian literature. Essays in honour of J. C. H. Lebram, Leiden, S. 202-249.

Dakhlia, Jocelyne (2008): Lingua Franca. Histoire d'une langue métisse en Méditerranée, Arles.

Dakhlia, Jocelyne (2010): »Histoire de la lingua franca«, in: Dervin, Fred (Hg.): Lingua Francas. La Véhicularité linguistique pour vivre, travailler et étudier, Paris, S. 21-27.

de Libera, Alain (2008): »Landerneau terre d'Islam«, Télérama.fr, 28.04.2008, www.telerama.fr/ idees/landerneau-terre-d-islam-par-alain-de-libera,28252.php, heruntergeladen am 19.11.2010.

Droit, Roger-Pol (2008): »Et si l'Europe ne devait pas ses savoirs à l'islam?«, Le Monde, 04.04.2008, S. 6 .

Durchhardt, Heinz / Kunz, Andreas (Hg.) (1997): `Europäische Geschichter als historiographisches Problem, Mainz.

Ellis, Roger (Hg.) (1989-2009): The Medieval Translator, Turnhout.

Endreß, Gerhard (Hg.) (1989/1997): Akten des Symposium Graeco-Arabicum, 2 Bde., Amsterdam.

Epstein, Steven A. (2006): Purity Lost. Transgressing Boundaries in the Eastern Mediterranean, 10001400, Baltimore.

Flaig, Egon (2006): »Der Islam will die Welteroberung «, Frankfurter Allgemeine Zeitung, Nr. 216, 16.09.2006, S. 35 .

Ford, Peter (2001): »Europe Cringes at Bush \Crusade` Against Terrorists «, The Christian Science Monitor, 19.11.2001, unter: www.csmonitor.com/2001/0919/p12s2-woeu.html, heruntergeladen am 05.05.2007.

Fossier, Robert (2000): Les relations des pays d'Islam avec le monde latin: du milieu du $X^{e}$ au milieu du XIII siècle, Paris.

Foz, Clara (2000): El traductor, la iglesia y el rey: la traducción en España en los siglos XII y XIII, Barcelona.

FranceMed (R. Abdellatif, Y. Benhima, D. König, E. Ruchaud) (2011): »Introduction à l'étude des transferts culturels en Méditerranée médiévale«, in: FranceMed (Hg.): Construire la Méditerranée, penser les transferts culturels. Approches historiographiqes et perspectives de recherche, München. 
FranceMed (R. Abdellatif, Y. Benhima, D. König, E. Ruchaud) (Hg.) (im Druck): Acteurs des transferts culturels en Méditerranée médiévale, München.

Gli Ebrei e le Scienze / The Jews and the Sciences = Micrologus 9 (2001).

Gouguenheim, Sylvain (2008): Aristote au Mont Saint-Michel. Les racines grecques de l'Europe chrétienne, Paris.

Graf, Georg (1949): Geschichte der christlichen arabischen Literatur, Bd. 3, Città del Vaticano.

Graf, Georg (1951): Geschichte der christlichen arabischen Literatur, Bd. 4, Città del Vaticano.

Guichard, Pierre / Sénac, Philippe (2000): Les relations des pays d'Islam avec le monde latin: milieu $X^{e}-$ milieu XIII ${ }^{e}$ siècle, Paris/Poitiers.

Gutas, Dimitri (1998): Greek Thought, Arabic Culture. The Greco-Arabic Translation Movement in Baghdad and Early 'Abbāsid Society (2nd-4th/8th-10th centuries), New York.

Hagemann, Ludwig (1985): »Die erste lateinische Koranübersetzung - Mittel zur Verständigung zwischen Christen und Muslimen im Mittelalter?«, in: Zimmermann, Albert / CraemerRuegenberg, Ingrid (Hg.): Orientalische Kultur und europäisches Mittelalter, Berlin / New York, S. 4558.

Hasse, Dag Nikolaus (2006): »The Social Conditions of the Arabic-(Hebrew-)Latin Translation Movements in Medieval Spain and in the Renaissance«, in: Speer / Wegener (2006), S. 68-88. Hasse, Dag Nikolaus (2008): »Pseudowissenschaft vom Abendland«, in: Frankfurter Allgemeine Zeitung, Nr. 141, 19.06.2008, S. 40.

Hershon, Cyril (2009): »Les ibn Tibbon dynastie de traducteurs«, in: Kappler / Thiolier-Méjean (Hg.): Le Plurilinguisme, S. 123-132.

Hiḍ̂r, al- 'Ádil (2008): Fī ǧadal ḥawla kitāb Arisțū fī ğabal Sān Mišāl. Ayya mawqif nattahuud min al-ḩițāb al-histīīi [Über die Debatte um das Buch Aristoteles auf dem Mont-Saint-Michel. Welche Haltung wir gegenüber dem hysterischen Diskurs einnehmen], al-Awān, 17. Dezember 2008, http://www.alawan.org/\%D9\%81\%D9\%8A-\%D8\%A7\%D9\%84\%D8\%AC\%D8\%AF\%D9\%84\%D8\%AD\%D9\%88\%D9\%84-\%D9\%83\%D8\%AA\%D8\%A7\%D8\%A8.html, heruntergeladen am 13.05.2011.

Hunke, Sigrid (1960): Allahs Sonne über dem Abendland. Unser arabisches Erbe, Stuttgart.

Jankrift, Kay Peter (2008): »Rechtsgeschäfte, Handelsalltag und die übersetzte Stimme des Herrn. Dolmetscher im Zeitalter der Kreuzzüge«, in: Moos (Hg.): Zwischen Babel und Pfingsten, S. 477-484. Jansen, Philippe / Nef, Annliese / Picard, Christophe (2000): La Méditerranée entre pays d'Islam et monde latin, Paris.

Jehel, Georges (1996): »Jews and Muslims in Medieval Genoa: From the Twelfth to the Fourteenth Century«, in: Arbel, Benjamin (Hg.): Intercultural Contacts in the Medieval Mediterranean, London / Portland, S. 120-132.

Jehel, Georges / Jehel, Simone (2000): Les relations des pays d'Islam avec le monde latin: milieu $X^{e}-$ milieu XIII siècle. Textes et documents, Paris.

Johns, Jeremy (2002): Arabic Administration in Norman Sicily: The Royal dīwān, Cambridge / New York.

Kappler, Claire / Thiolier-Méjean, Suzanne (Hg.) (2009): Le Plurilinguisme au Moyen Âge. OrientOccident, Paris. 
Kedar, Benjamin Z. / Aslanov, Cyril (2009): »Problems in the Study of Trans-Cultural Borrowing in the Frankish Levant«, in: Borgolte / Schneidmüller (Hg.): Hybride Kulturen, S. 277-286.

Klinge, G. (1939): »Die Bedeutung der syrischen Theologen als Vermittler der griechischen Philosophie an den Islam«, Zeitschrift für Kirchengeschichte, 58, S. 346-386.

König, Daniel (2006): »Der Nutzen von Außenperspektiven. Das europäische Mittelalter aus moderner arabischer Perspektive«, in: Conermann, Stephan / Heinze, Marie-Christine (Hg.): Bonner Islamwissenschaftler stellen sich vor, Schenefeld, S. 197-242.

König, Daniel (2008): »Wie eine Religion Staat und Gesellschaft durchdringt. Zu Entstehungsbedingungen und -voraussetzungen einer staats-, gesellschafts- und kulturtragenden Religion«, in: Heidenreich, Felix / Merle, Jean-Christophe / Vogel, Wolfram (Hg.): Staat und Religion in Frankreich und Deutschland / L'Etat et la religion en France et en Allemagne, Berlin, S. 12-62.

König, Daniel (2010a): »Muslim Perception(s) of Latin-Christianity. Methodological Reflections and a Reevaluation«, Comparativ. Zeitschrift für Globalgeschichte und vergleichende Gesellschaftsforschung, 20(4), S. 18-42.

König, Daniel (2010b): »Zur Ausstrahlung des Papsttums in die mittelalterliche arabischislamische Welt. Eine Evaluation der arabisch-islamischen Berichterstattung zum Bischof von Rom«, Quellen und Forschungen aus italienischen Archiven und Bibliotheken, 90, S. 1-52.

Kritzeck, James (1964): Peter the Venerable and Islam, Princeton.

Kybeline (2008), unter: www.kybeline.com/2008/05/01/sylvain-gouguenheim-der-islam-spieltekeine-rolle, 01.05.2008, heruntergeladen am 19.11.2010.

L'Express.fr (2008): »Le Goff défend Gouguenheim «, L'Express.fr., 15.05.2008, www.lexpress.fr/ informations/le-goff-defend-gouguenheim_723012.html, heruntergeladen am 19.11.2010.

Le Point (2011), »Croisade islamiste en Egypte, Irak, au Pakistan ... La chasse aux chrétiens«, Le Point, $\mathrm{n}^{\circ}$ 1999, 06.01.2011.

Le Conservateur (2008), unter: http://leconservateur.bafweb.com/index.php?2008/04/30/1336il-faut-soutenir-sylvain-gouguenheim, 30.04.2008, heruntergeladen am 19.11.2010.

Lejbowicz, Max (Hg.) (2008): L'Islam médiéval en terres chrétiennes. Science et idéologie, Villeneuve d'Ascq.

Le Salon Beige (2008), unter: www.lesalonbeige.blogs.com/my_weblog/2008/04/fatwa-contresy.html, 30.04.2008, heruntergeladen am 19.11.2010.

Levi della Vida, Giorgio (1954): »La Traduzione araba delle storie di Orosio«, Al-Andalus, 19, S. 257-293.

Lewis, Bernard (1982/2001): The Muslim Discovery of Europe, New York.

Lupprian, Karl-Ernst (1981): Die Beziehungen der Päpste zu islamischen und mongolischen Herrschern im 13. Jh. anhand ihres Briefwechsels, Città del Vaticano.

Mas Latrie, Louis de (1866/1872): Traités de paix et de commerce et documents divers concernant les relations des Chrétiens avec les Arabes de l'Afrique septentrionale au Moyen Age, 2 Bde., Paris.

Maser, Matthias (2006): Die Historia Arabum des Rodrigo Jiménez de Rada. Arabische Traditionen und die Identität der Hispania im 13. Jahrhundert, Berlin, S. 187-193.

Maser, Matthias (2007): »Übersetzung und Identität«, in: Herbers, Klaus / Jaspert, Nikolas (Hg.): Grenzräume und Grenzüberschreitungen im Vergleich. Der Osten und der Westen des mittelalterlichen Lateineuropa, Berlin, S. 241-260. 
Mazzoli-Guintard, Christine / Martínez Enamorado, V. / Viguera Molins, Maria Jesús (1999): Les Relations des pays d'Islam avec le monde latin: milieu $X^{e}-$ milieu XIII ${ }^{e}$ siècle. Analyse et synthèse, Paris.

Meier, Christian (2009): Kultur um der Freiheit willen. Griechische Anfänge - Anfänge Europas?, München.

Mersch, Margit / Ritzerfeld, Ulrike (Hg.) (2009): Lateinisch-griechisch-arabische Begegnungen. Kulturelle Diversität im Mittelmeerraum des Spätmittelalters, Berlin.

Metcalfe, Alex (2003): Muslims and Christians in Norman Sicily. Arabic Speakers and the End of Islam, London / New York.

Millet-Gérard, Dominique (1984): Chrétiens mozarabes et culture islamique dans l'Espagne des VIII ${ }^{e}-I^{e}$ siècles, Paris.

Minervini, Laura (1996): »Les contacts entre indigènes et croisés dans l'Orient latin: le rôle des drogmans«, in: Lüdtke, Jens / Kontzi, Reinhold (Hg.): Romania Arabica, Tübingen, S. 57-62.

Molina, Luis (1984): »Orosio y los geógrafos hispanomusulmanes», al-Qantara, 5, S. 63-92.

Moos, Peter von (Hg.): Zwischen Babel und Pfingsten. Sprachdifferenzen und Gesprächsverständigung in der Vormoderne (8.-16. Jh.) / Entre Babel et Pentecôte. Différences linguistiques et communication orale avant la modernité (VIII ${ }^{e}$ XVI ${ }^{e}$ siècle), Wien / Berlin.

Musall, Frederek (2008): „Vom `Schlüssel der Wissenschaften` zum `Schlüssel des Gesetzes`. Wissenskultur und Wissenstransfer im europäischen Mittelalter am Beispiel Mosche ben Maimons«, in: Borgolte, Michael / Schiel, Juliane / Schneidmüller, Bernd / Seitz, Annette (Hg.): Mittelalter im Labor. Die Mediävistik testet Wege zu einer transkulturellen Europawissenschaft, Berlin, S. 210-228.

O'Leary, De Lacy (2001): How Greek Science Passed to the Arabs, London / New York.

Oschema, Klaus (2006): »Europa in der mediävistischen Forschung - eine Skizze«, in: Schwinges, Rainer / Hesse, Christian / Moraw, Peter (Hg.): Europa im späten Mittelalter, München, S. 11-32. Osman, Nabil (2010): Kleines Lexikon deutscher Wörter arabischer Herkunft, 8. Aufl., München. Ouerfelli, Mohamed (2008): Le sucre. Production, commercialisation et usages dans la Méditerranée médiévale, Leiden.

Penelas, Mayte (2001): »A Possible Author of the Arabic Translation of Orosius' Historiae«, alMasaq, 13, S. 113-135.

Penelas, Mayte (2006): »Linguistic Islamization of the `Mozarabs as Attested in a Late NinthCentury Chronicle«, in: Bremer, Ernst / Jarnut, Jörg / Richter, Michael / Wasserstein, David J. (Hg.): Language of Religion - Language of the People. Medieval Judaism, Christianity and Islam, München, S. 103-114.

Petition ENS LSH (2008) : " Pétition de l'École normale supérieure Lettres et sciences humaines ", in: www.telerama.fr/idees/petition-de-l-ecole-normale-superieure-lettres-et-sciences-humaines, 28371.php, heruntergeladen am 19.11.2010.

Rdv-histoire (2008), unter: www.rdv-histoire.com/fichiers/pdf/journal_2008.pdf.

Ricklin, Thomas (2006): ")Arabes contigit imitari<. Beobachtungen zum kulturellen Selbstverständnis der iberischen Übersetzer der ersten Hälfte des 12. Jahrhunderts«, in: Speer / Wegener (2006), S. 47-67.

Riley-Smith, Jonathan (1972): „Some Lesser Officials in Latin Syria», The English Historical Review, 87, S. 1-26. 
Rosenthal, Franz (1975): The Classical Heritage in Islam, Berkeley / Los Angeles.

Rudolph, U. (2004): »Les traductions arabes des œuvres d'Augustin«, in: Bouayed, F. Z. (Hg.): Le Philosophe algérien. Saint Augustin, africanité et universalité, Alger, S. 129-134.

Ruggles, D. Fairchild (2004): »Mothers of a Hybrid Dynasty. Race, Genealogy, and Acculturation in al-Andalus «, Journal of Medieval and Early Modern Studies, 34(1), S. 65-94.

Salama-Carr, Myriam (1990): La traduction à l'époque abbasside: l'école de Hunayn Ibn Isḥāq et son importance pour la traduction, Paris.

Sarmiento Pérez, Marcos (2008): Cautivos qui fueron intérpretes. La comunicación entre europeos, aborígenes canarios y berberiscos durante la conquista de Canarias y los conatos en el Norte de África (1341-1569), Málaga.

Schiel, Juliane / Schneidmüller, Bernd / Seitz, Annette (2009): »Hybride Kulturen im mittelalterlichen Europa«, in: Borgolte, Michael / Schneidmüller, Bernd (Hg.): Hybride Kulturen, S. 9-24.

Schipperges, Heinrich (1955): „Die frühen Übersetzer der arabischen Medizin in chronologischer Sicht«, Sudhoffs Archiv für die Geschichte der Medizin, 39, S. 53-93.

Schipperges, Heinrich (2006): Selected Writings on the Reception and Assimilation of Islamic Medicine in the Occident, ed. Fuat Sezgin, Frankfurt a. M.

Sénac, Philippe (2000): La Frontière et les hommes (VIII - XII ${ }^{e}$ siècle). Le peuplement musulman au nord de l'Ebre et les débuts de la reconquête aragonaise, Paris.

Sezgin, Fuat (Hg.) (2005): The Reception and Assimilation of Arabic Science in the Occident, 6 Bde., Frankfurt a. M.

Sezgin, Fuat (Hg.) (2005): The Reception and Assimilation of Greek and Indian Science in Islam, 4 Bde., Frankfurt a. M.

Sezgin, Fuat (Hg.) (2005-06): The Reception and Assimilation of Islamic Medicine in the Occident, 2 Bde., Frankfurt a. M.

Sezgin, Fuat (Hg.) (2006): Leonardo Fibonacci and his Arabic Sources, Frankfurt a. M.

Sezgin, Fuat (Hg.) (2006): The Banû Mûsâ and Thâbit ibn Qurra. Their Works in Western Translations and Adaptations, Frankfurt a. M.

Sezgin, Fuat (Hg.) (2006): Al-Kitâb al-mukhtasar fî hisâb al-jabr wa-l-muqâbala by Muhammad ibn Mûsâ al-Khwârizmî. Western Translations and Adaptations, Frankfurt a. M.

Sezgin, Fuat (Hg.) (2006): Az-Zarqâlî and al-Bitrûjî. Their Works in Western Translations and Adaptations, Frankfurt a. M.

Sezgin, Fuat (Hg.) (2006): Constantinus Africanus and Arabic Medicine. The School of Salerno, 3 Bde., Frankfurt a. M.

Sezgin, Fuat (Hg.) (2006): Mâshâ'allâh and al-Farghânî. Their Works in Western Translations, Frankfurt a. M.

Sezgin, Fuat (Hg.) (2006): The Astronomical Works of Thabit b. Qurra by Francis J. Carmody, Frankfurt a. M.

Sezgin, Fuat (Hg.) (2006): The Book on Arithmetic by Muhammad Ibn Mûsâ al-Khwârizimî. Western Translations and Adaptations, 2 Bde., Frankfurt a. M. 
Sezgin, Fuat (Hg.) (2006): The Reception and Assimilation of Arabic Medicine. The School of Montpellier, Frankfurt a. M.

Sezgin, Fuat (Hg.) (2006): The Reception and Assimilation of Islamic Mathematics and Astronomy in the Occident, 4 Bde., Frankfurt a. M.

Sezgin, Fuat (Hg.) (2007): Arabic Versions of Aristotle's Works in Latin Translation, 2 Bde., Frankfurt a. M.

Sezgin, Fuat (Hg.) (2007): Averroes Latinus, Frankfurt a. M.

Sezgin, Fuat (Hg.) (2007): Avicenna Latinus. The Reception and Assimilation of Ibn Sînâ in the West, 2 Bde., Frankfurt a. M.

Sezgin, Fuat (Hg.) (2007): Gerbert and the Transmission of the Arabic-Islamic Sciecnes to the Occident, 2 Bde., Frankfurt a. M.

Sezgin, Fuat (Hg.) (2007): Kitâb al-Anwâ' by 'Arîb ibn Sa'd al-Qurtubî and its Latin Translations, Frankfurt a. M.

Sezgin, Fuat (Hg.) (2007): The Hindu-Arabic Numerals and their Transmission to the Occident, Frankfurt a. M.

Sezgin, Fuat (Hg.) (2007): The Optics of Ptolemy and its Arabic-Latin Transmission, Frankfurt a. M. Sezgin, Fuat (Hg.) (2007): The Reception of Islamic Philosophy in the Latin West, Frankfurt a. M. Sezgin, Fuat (Hg.) (2007): The Tables of al-Khwârizmî and the Tabulae Toletanae, Frankfurt a. M. Sezgin, Fuat (Hg.) (2007): Two Texts by Ibn Rushd in their Medieval Latin Translation, Frankfurt a. M. Shalem, Avinoam (1998): Islam Christianized. Islamic Portable Objects in the Medieval Church Treasuries of the Latin West, Frankfurt a. M.

Sibon, Juliette (im Druck): Les Juifs de Marseille au XIV siècle, Paris.

Sivo, Vito (1995): »Lingue e interpreti«, in: Musca, Giosuè (Hg.): Strumenti, tempi e luoghi di comunicazione nel Mezzogiorno normanno-svevo, Bari, S. 89-111.

Speer, Andreas / Wegener, Lydia (Hg.) (2006): Wissen über Grenzen. Arabisches Wissen und lateinisches Mittelalter, Berlin / New York.

Spuler, Berthold (1980): »Hellenistisches Denken im Islam«, in: Id.: Gesammelte Aufsätze, Leiden, S. 13-26.

Steinschneider, Moritz (1893): Die hebräischen Übersetzungen des Mittelalters und die Juden als Dolmetscher, Berlin.

Strohmaier, Gotthard (2003): »Die Griechen waren keine Europäer«, in: Id.: Hellas im Islam. Interdisziplinäre Studien zur Ikonographie, Wissenschaft und Religionsgeschichte, Leipzig, S. 1-6.

Talīlī, Ḥassān (2008): »muḥāwalat li-tamhīš faḍl al-ḥaḍāra al-'arabiyya al-islāmiyya 'alā 'l-ğarb« [»Ein Versuch, die Überlegenheit der arabisch-islamischen Zivilisation gegenüber dem Westen zu minimieren«], ar-Riyāọ, Nr. 14569, 14.05.2008, www.alriyadh.com/2008/05/14/ article342466.html, heruntergeladen am 19.11.2010.

Tazi, Raja (1998): Arabismen im Deutschen. Lexikalische Transferenzen vom Arabischen ins Deutsche, Berlin / New York.

Teixidor, Javier (2001): „D’Antioche à Bagdad. Bibliothèques et traductions syriaques«, in: Giard, Luce / Jacob, Christian (Hg.): Des Alexandries. Du livre au texte, Paris, S. 249-262. 
Teixidor, Javier (2003): Aristote en syriaque. Paul le Perse, logicien du VIe siècle, Paris.

Teixidor, Javier (2007): Hommage à Bagdad: traducteurs et lettrés de l'époque abbaside, Paris.

Tolan, John (2002): Saracens. Islam in the Medieval European Imagination, New York.

Tolan, John (2008): »Porter la bonne parole auprès de Babel. Les problèmes linguistiques chez les missionnaires mendiants, XIII ${ }^{\mathrm{e}}-\mathrm{XIV}{ }^{\mathrm{e}}$ siècle«, in: Moos (Hg.): Zwischen Babel und Pfingsten, Wien / Berlin, S. 533-548.

Tolan, John / Josserand, Philippe (2000): Les relations des pays d'Islam avec le monde latin: milieu $X^{e}$ milieu XIII ${ }^{e}$ siècle, Rosny-sous-Bois.

Troupeau, Gérard (1991): „Le rôle des syriaques dans la transmission et l'exploitation du patrimoine philosophique et scientifique grec«, Arabica, 38, S. 1-10.

Tyan, Emile (1960): Histoire de l'organisation judiciaire en pays d'Islam, 2. überarb. Aufl., Leiden.

Unger, Andreas (2006): Von Algebra bis Zucker. Arabische Wörter im Deutschen, München.

Vernet, Juan (1978): La cultura hispanoárabe en Oriente y Occidente, Barcelona.

Vernet, Juan (1999): Lo que Europa debe al Islam de España, Barcelona.

Vinocur, John (2008): „Europe's Debt to Islam Given a Skeptical Look«, International Herald Tribune, 28.04.2008, www.nytimes.com/2008/04/28/world/europe/28iht-politicus.

2.12398698.html, heruntergeladen am 15.11.2010.

Volpi, Franco (2009): »Aristotele e l'Islamofobia«, La Repubblica, 11.03.2009, S. 39.

Walzer, Richard (1962): Greek into Arabic. Essays on Islamic Philosophy, Oxford.

Wansbrough, John (1961): Documents for the History of Commercial Relations Between Egypt and Venice 1442-1512, PHD diss., University of London.

Wansbrough, John (1996): Lingua Franca in the Mediterranean, Richmond.

Wetzel, Johannes (2008): »Was Europa dem Islam verdankt - und was nicht«, Welt-Online, http:// www.welt.de/kultur/article1957436/Was_Europa_dem_Islam_verdankt_und_was_nicht.html, heruntergeladen am 13.05.2011.

Wohlmuth, Joseph (Hg.) (2000): Konzilien des Mittelalters. Vom ersten Laterankonzil (1123) bis zum fünften Laterankonzil (1512-1517), Paderborn.

Wright, Roger (1982): Late Latin and Early Romance in Spain and Carolingian France, Liverpool.

Zonta, Mauro (2006): »The Jewish Mediation in the Transmission of Arabo-Islamic Science and Philosophy to the Latin Middle Ages. Historical Overview and Perspectives of Research«, in: Speer / Wegener (2006), S. 89-105.

\section{NOTES}

1. Ibn Ḩaldūn, tārīh, ed. Zakkār / Šaḥāda, Bd. 1, S. 629, übers. Daniel König.

2. Dieses Wissen wurde auch bewusst gefördert. So etwa hatte 2001 in Frankreich die staatliche Prüfung zur Erteilung der Lehrerlaubnis an Sekundarschulen und Universitäten (CAPES, Agrégation) im Bereich Geschichte und Geographie die Beziehungen zwischen der islamischen Welt und dem lateinischen Westen zwischen dem 10. und 13. Jahrhundert zum Thema. Dies zog eine Reihe von studiumsrelevanten Veröffentlichungen nach sich. Siehe Mazzoli-Guintard / Martínez Enamorado / Viguera Molins (1999); CIHAM (2000); Guichard / Sénac (2000); Sehel / 
Sehel (2000); Jansen / Nef / Picard (2000); Tolan / Josserand (2000); Fossier (2000); Balard / Bresc / Durand (2000); Aillet / Makariou / Tixier-Caceres / Gourdin / Martinez-Gros (2001); Arrignon / Bousquet-Labouérie, Leroy (2001).

3. Siehe etwa kürzlich die Ausgabe des französischen Politmagazins »Le Point« (2011) zum Thema "Croisade islamiste en Egypte, Irak, au Pakistan ... La chasse aux chrétiens«.

4. Bakr / Ezbidi / Kassab-Hassan / Karcic / Zaidi / Jawhar Hassan (2004), S. 27 f.; König (2006),

S. 221-224.

5. Vgl. Ford (2001).

6. König (2008), S. 25-36.

7. Bawer (2006), S. 104, in einem Kapitel unter dem Titel »Europe's Weimar movement«.

8. Gouguenheim (2008), S. 17.

9. Droit (2008), S. 6.

10. Petition ENS LSH (2008).

11. Collectif international (2008).

12. al-Ḥayāt (2008).

13. Deren Ergebnis ist eine Gegenschrift, die u. a. eine Liste der frankophonen Reaktionen auf das Werk bis zum 31.12.2008 enthält: Lejbowicz (2008), S. 30-44.

14. Rdv-histoire (2008).

15. Colloque L'Islam et l'Occident (2009).

16. Vinocur (2008); Wetzel (2008); Hasse (2008), S. 40; Ben Jelloun (2008), S. 56; Volpi (2009), S. 39; Talīìi (2008).

17. Lejbowicz (2008); Büttgen / de Libera / Rached / Rosier-Catach (2009).

18. L'Express.fr (2008).

19. Le Conservateur (2008); Le Salon Beige (2008); Kybeline (2008).

20. de Libera (2008).

21. Siehe hierzu die Interpretation des arabischen Internetmagazins al-Awān in dem Artikel von Hiḍr (2008).

22. Hierzu: Strohmaier (2003); Durchhardt / Kunz (1997); Oschema (2006), dessen derzeitiges Forschungsprojekt »Bilder von Europa. Europa-Konzepte im Mittelalter und in der mediävistischen Forschung «, bald weitere Ergebnisse erzielen wird.

23. Gouguenheim (2008), S. 55: "aspiration de l'esprit européen à une pensée libre«, S. 73, S. 138, S. 164, S. 176: "une autre originalité de l'esprit grec, si présente encore de nos jours en Occident, l'esprit critique et l'exercice de l'autocritique«, S. 198.

24. Meier (2009), S. 7-60.

25. Der Althistoriker Egon Flaig stellt Islam und »freies griechisches Denken« als Gegensatzpaar dar, siehe: Flaig (2006), S. 35.

26. Gouguenheim (2008), S. 75-101, S. 125-166.

27. Gouguenheim (2008), S. 25-73.

28. Gouguenheim (2008), S. 125-166.

29. Gouguenheim (2008), S. $198 \mathrm{f}$.

30. Hunke (1960); Vernet (1999); ursprünglich veröffentlicht als Vernet (1978).

31. Sezgin (2005-2007); Schipperges (2006).

32. $\mathrm{Zu}$ diesen zählen etwa Baldassarre Boncompagni, Marie-Thérèse d'Alverny, Douglas Morton Dunlop, Johann Fück, Francesco Gabrieli, Ignaz Goldziher, Gustav Edmund von Grunebaum, Charles Homer Haskins, Ramón Menéndez Pidal, José María Millás Vallicrosa, Carlo Alfonso Nallino, Delacy O'Leary, Rudi Paret, Claudio Sánchez-Albornoz, Joseph Schacht, Heinrich Schipperges, Berthold Spuler, Moritz Steinschneider, Karl Sudhoff, Lynn Thorndike, Richard Walzer, Gaston Wiet, Ferdinand Wüstenfeld und viele andere.

33. $\mathrm{Zu}$ ihnen zählen - um nur einige zu nennen - Dionisius Agius, Charles Burnett, Peter Dilg, Gerhard Endreß, Menso Folkerts, Dimitri Gutas, Dag Nikolaus Hasse, Donald Hill, Danielle 
Jacquart, Felix Klein-Franke, Paul Kunitzsch, David Lindberg, Thomas Ricklin, Gotthard Strohmaier, Javier Teixidor usw.

34. Steinschneider (1893); Gli Ebrei e le Scienze (2001); Zonta (2006); Musall (2008); Sibon (im Druck).

35. Attias (2009).

36. Borgolte / Schiel (2008), S. 16 f; Schiel / Schneidmüller / Seitz (2009), S. 10.

37. Kedar / Aslanov (2009). Interessant ist v. a. das Phänomen der spätestens seit dem Spätmittelalter im Mittelmeerraum gebräuchlichen `Lingua Franca‘, hierzu Dakhlia (2008).

38. Siehe etwa Shalem (1998); Mersch / Ritzerfeld (2009).

39. Corriente (2008).

40. Unger (2006); Osman (2010). Ausführlicher auf sprachwissenschaftlicher Grundlage: Tazi (1998).

41. Hierzu das Kapitel »Objets - espaces - acteurs« in: FranceMed (2011).

42. Ouerfelli (2008).

43. Tolan (2002); König (2010a), mit weiterer Literatur auf S. 20, Anm. 9.

44. Ruggles (2004); Christ (2006); Epstein (2006); FranceMed (im Druck).

45. Hierzu das Kapitel »Objets - espaces - acteurs« in: FranceMed (2011).

46. Beer (1989); Ellis (1989-2009).

47. Exemplarisch für diese Tendenz steht etwa Walzer (1962). Sie wird in der neueren Forschung auch weitergeführt, vgl. Endreß (1989/1997).

48. O'Leary (2001), S. 155-175; Salama-Carr (1990); Gutas (1998), bes. S. 136-141; Teixidor (2007).

49. Klinge (1939), S. 346-386; Spuler (1980), S. 13-26; Troupeau (1991); Teixidor (2001); Teixidor (2003).

50. Zu möglichen Quellen der arabisch-islamischen Historiographen siehe die sehr ausführlichen Angaben des Gelehrten al-Mas' ūdī, kitāb at-tanbīh wa 'l-išrāf, ed. de Goeje, S. 154 f. Übersetzt als: Maçoudi, Livre de l'avertissement, übers. Carra de Vaux, S. 212 f. Vgl. auch König (2010b), § 1a, § $2 \mathrm{a}$.

51. Rosenthal (1975), S. 10; Gutas (1998), S. 193-196.

52. Schipperges (1955), S. 53-93; Ricklin (2006); Hasse (2006); Burnett (2009); Foz (2000).

53. Neben den in Anm. 34 genannten Werken vgl. Cohn (1935); Aslanov (2009); Hershon (2009).

54. Hagemann (1985); Burman (2009).

55. Crónica del moro Rasis, ed. Catalán, de Andrés.

56. Maser (2006), S. 187-193.

57. Maser (2007), S. 241-260.

58. Siehe z. B. Millet-Gérard (1984), S. 27 f., S. 53-62, S. 68-70, S. 71-76; Burman (1994), S. 78, 157, 175, 194 f.; Aillet / Penelas / Roisse (2008).

59. Siehe z. B. Penelas (2006); Wright (1982), S. 151-162.

60. Le psautier mozarabe de Hafs le Goth, ed. Urvoy, S. IV-V.

61. Siehe hierzu die Erörterungen von Levi della Vida (1954), S. 257-293; Badawī (1982), S. 10-17; Daiber (1986); kitāb Hurūšiyūš, ed. Penelas, S. 27-42 (Einleitung); Penelas (2001).

62. Penelas (2006), S. 106, Anm. 17.

63. kitāb Hurūšiyūǔs, ed. Penelas, S. 16 (Arabischer Text), S. 47-66, S. 99-119 (Einleitung); Penelas (2006), S. 106; Crónica del moro Rasis, ed. Catalán / de Andrés, S. LIX (Einleitung); Molina (1984), S. 91.

64. al-Mas 'ūdī, murūğ a $\underline{d}$-d dahab, § 912, ed./übers. Barbier de Ménard / Pavet de Courteille / Pellat, S. 146 (Arab.), S. 344 (Franz.).

65. Graf (1949), S. 216-220, 347-349, 495; Graf (1951), S. 41, 47-49, 160-162, 232, 236, 256; Rudolph (2004), S. 129-134.

66. Sivo (1995).

67. Riley-Smith (1972); Minervini (1996); Bosselmann-Cyran (1997); Jankrift (2008).

68. Tolan (2008). 
69. Sarmiento Pérez (2008).

70. Vgl. z. B. Annales regni Francorum, a. 801, ed. Pertz / Kurze, S. 116.

71. Ibn Hurradaḍbih, kitāb al-masālik, ed. de Goeje, S. 153 f. Hierzu: Ashtor (1977).

72. Iohannes abbas Sancti Arnulfi, Vita Iohannis Gorziensis, § 115-136, ed. Pertz, S. 369-377, bes. § 121, S. 371, und § 128, S. 374; Ibn 'Iḍārī, al-bayān al-mugirib, ed. Colin / Lévi-Provençal, Bd. 2, S. 218; Ibn Haldūn, tārīh, ed. Zakkār / Šaḥāda, Bd. 4, S. 183.

73. Zum Phänomen der Grenzregionen siehe u. a. die Untersuchungen zur Iberischen Halbinsel von Sénac (2000); Buresi (2004).

74. Chronicon Salernitanum, § 110-111, ed. Westerbergh, S. $122 \mathrm{f}$.

75. Ademarus Cabannensis, Chronicon (recensiones beta et gamma), III,52, ed. Bourgain / Landes / Pon, S. 171.

76. Ibn Ğubayr, riḥla, kein Editor, S. 271, S. 275, S. 277-279.

77. Ibn Ğubayr, riḥla, kein Editor, S. 283. Der Begriff wird mit »huğğḡăğ bayt al-maqdis « übersetzt.

78. Dakhlia (2010), S. 22 f; Dakhlia (2008), S. 16 f.

79. Johns (2002), S. 207, S. 297 f. Zum weiteren trilingualen Phänomenen in Sizilien: Metcalfe (2003), S. 127 f., zum trilingualen administrativen Vokabular besonders S. 135-137.

80. Siehe z. B. die Dokumente in: Mas Latrie (1866/1872); Wansbrough (1961); Burns / Chevedden (1999).

81. Vgl. Siete Partidas, ed. Real Academia, Bd. 3, partida quinta, capitulo XI, ley I, S. 255. Englische Übersetzung: Las Siete Partidas, ed. Burns, übers. Scott, Bd. 4, S. 1092.

82. Riley-Smith (1977), S. 15-19.

83. Siehe Tyan (1960), S. 72, S. 258, mit Angaben zum Amt des Übersetzers (mutarğim) in Rechtsgeschäften als Gehilfe des Richters (al-qāọi), dessen Anwesenheit schon in den frühen Jahrhunderten des islamischen Rechts als erforderlich erachtet wird.

84. al-Qalqašandī, kitāb șubḥ al- 'ašă', ed. Ibrāhīm, Bd. 5, S. 304. Englische Übersetzung und Kommentar in: Wansbrough (1996), S. 78.

85. Siehe den Vertrag bei Mas Latrie (1866), Bd. 1, S. 189, S. 192.

86. Concilium Viennense (a. 1311-1312), decretum 24, ed./übers. Alberigo / Wohlmuth, in: Wohlmuth (2000), Bd. 2, S. 379.

87. Matthaeus Parisiensis, chronica majora, ed. Luards, Bd. 4, anno 1246, S. 566-568; Matthaeus Parisiensis, Historia Anglorum, ed. Madden, Bd. 3, S. 11; Radulfus de Diceto, Opera historica, ed. Stubbs, Bd. 2, S. 25 f. Vgl. auch die lateinischen Übersetzungen arabischer Briefe an den Papst bei Lupprian (1981).

88. Altaner (1933), S. 233-241; Burman (2007); Tolan (2008), S. 533-548.

89. Lewis (1982/2001), S. 81, S. 105.

90. Petrus Venerabilis, Contra sectam Saracenorum, ed. Kritzeck, in: Kritzeck (1964), S. 229; Cronica del moro Rasis, ed. Catalan / de Andres, S. XI, S. 3 mit Anm. 1-3.

91. Mas Latrie (1886), Bd. 1, S. 205, S. 365 f.

92. Canale (1860), S. 352; Jehel (1996), S. 123.

93. Mas Latrie (1886), Bd. 1, S. 220 f., S. 237, S. 249.

94. Mas Latrie (1866), Bd. 1, S. 350. 